\title{
Out of the dark, into the light: sexuality and fertility in pediatric urological conditions
}

\author{
Bernhard Haid $\mathbb{1}^{1,2} \cdot$ Mesrur Selçuk Silay $\mathbb{1}^{3,4}$
}

Received: 12 September 2020 / Revised: 10 January 2021 / Accepted: 21 January 2021 / Published online: 8 February 2021

(c) The Author(s), under exclusive licence to Springer Nature Limited 2021

Pediatric urologists are dealing with urogenital anomalies on a daily basis, often involving also the gonads and sometimes being part of very rare or syndromal conditions. Besides performing urogenital reconstructive surgery we aim to provide children a holistic care until adulthood (at least): Aspects of sexuality and fertility therefore must play an integral role in pediatric urology.

Yet, as most pediatric urologists mainly are treating children, with the larger part of the necessary (surgical) interventions being indicated and performed during young ages, sexuality as well as fertility might sometimes take a back seat in daily clinical routine. At the time, when these concerns become more important to the adolescent and young adult patients, they are also entering the critical and difficult phase of so-called "transition", often involving a disruption in care with a multitude of associated problems and again with a potential risk of not emphasizing sexuality and fertility enough $[1,2]$. Clearly, it is our responsibility to not allow for these important issues to get "lost in transition" (c Hettel [2]).

Inversely, the scope of pediatric sexual medicine is much larger than pediatric urology and not only touching to those we operate: the topics covered in the papers presented herein relate to pediatric endocrinology, psychology, and andrology as well as to rare topics and diseases in pediatric

Bernhard Haid

bernhard.haid@me.com

$\triangle$ Mesrur Selçuk Silay

selcuksilay@gmail.com

1 Department for Pediatric Urology, Ordensklinikum Linz, Hospital of the Sisters of Charity, Linz, Austria

2 Department of Urology, Ludwig-Maximilians University, Munich, Germany

3 Division of Pediatric Urology, Department of Urology, School of Medicine, Biruni University, Istanbul, Turkey

4 Istanbul Memorial Hospital, Istanbul, Turkey urology and surgery. This reflects how such problems often need to be addressed in an interdisciplinary way in order to obtain an optimal result for each patient.

In this special issue, we aimed to spotlight some aspects in the large spectrum of pediatric sexual medicine in conjunction with commonplace pediatric urological conditions as well as such being encountered once in a blue moon-with our premise being to give also space to (seemingly) marginal topics and out of the box perspectives.

A series of articles address outcomes of reconstructive surgical procedures and their underlying conditions:

While a circumcision might seem a banal intervention at first glance, it is worthwhile to review its potential consequences on the sexual life-with the review article by Beatriz Banuelos providing a broad overview [3].

In another narrative review, Murat Gul et al. provide a comprehensive outline of the literature on sexuality and fertility outcomes after hypospadias surgery highlighting clinically relevant insights as well as the inherent problems of the available data [4].

Celine Sinatti et al. are reporting about long-term sexuality outcomes in exstrophy patients-a diverse patient group with complex problems on the background of often numerous surgical interventions [5].

By means of an interview with experts in the field as well as a patient group representative, Beatriz Banuelos et al. tackle the sexual problems of patients who underwent augmentation surgery for neurogenic bladder emptying problems in childhood [6].

Only in rare cases, often in the context of complex urogenital malformations, where penile reconstruction to an extent allowing for adequate sexual function is not feasible, phalloplasties are the last resort for these boys and men: Celine Sinatti et al. looked back into their unique experience and report about long-term outcomes [7].

James Rague addresses the topic of testicular torsion with a focus on the danger for the loss of a testis and therefore a potential hazard to fertility [8]. 
Zinner's Syndrome is a rare diagnosis, however, its potential implications on fertility are real and should be addressed, as emphasized in the review article featuring a case study by Aybike Hofmann [9].

Sexuality also touches to indications of ritual, not medically indicated genital procedures in children: In order to address this topic from an uncommon angle, Brian Earp and colleagues discuss the potential implications -also for male circumcision-and the ethical background of the current female genital mutilation policy of the WHO [10].

One of the most difficult situations in daily clinical practice is a suspicion of a child having been sexually abused: Manuela Hiess is giving an overview including practical tips on how to approach these children best [11].

Gender dysphoria and gender diversity in children certainly poses a major challenge to not specialized clinicans: Karlien D'Hondt and colleagues provide a useful and most interesting insight into their experiences in this field with useful take outs that are applicable in clinical routine [12].

While the surgical treatment of children with differences of sex development (DSD) and its indications has been much discussed during the last years, their-and their families-needs in respect to psychosocial care and counseling are highlighted in a comprehensive article including a survey by Katinka Schweizer and colleagues [13]. The data on which this article is based on, date from 2015. Since then, the discussion about DSD evolved in many respects: these results and their discussion are not only an interesting read but also put the current efforts and controversies into perspective.

To summarize, this has turned out to become a very diverse collection of articles. In our opinion this might be a great opportunity to take a peek beyond one's own nose.

At last, it remains to acknowledge the dedicated and great work of all those writing, reviewing and processing these articles as well as to the members of the EAU Young Academic Urologists (YAU) working group on pediatric urology, who were involved in brainstorming potential topics as well as contributing articles.

Our gratitude belongs to Ege Can Serefoglu, the editor in chief of IJIR for his faith in our guest editorship and for providing us with the unique opportunity to compile this special issue and to Hodan Omar, who patiently assisted us during the process.

We sincerely hope you will enjoy reading it as much as we did editing.

\section{Compliance with ethical standards}

Conflict of interest The authors declare no competing interests.

Publisher's note Springer Nature remains neutral with regard to jurisdictional claims in published maps and institutional affiliations.

\section{References}

1. Harhuis A, Cobussen-Boekhorst H, Feitz W, Kortmann B. 5 years after introduction of a transition protocol: an evaluation of transition care for patients with chronic bladder conditions. J Pediatr Urol. 2018;14:150.e1-150.e5.

2. Hettel D, Tran C, Szymanski K, Misseri R, Wood H. Lost in transition: patient-identified barriers to adult urological spina bifida care. J Pediatr Urol. 2018;14:535.e1-535.e4.

3. Banuelos B, Leigh Garcia Hail J. Circumcision in childhood and male sexual function: a blessing or a curse? IJIR. 2020. https://doi. org/10.1038/s41443-020-00354-y. [Epub ahead of print].

4. Gul M, Hildorf S, Silay MS. Sexual functions and fertility outcomes after hypospadias repair. IJIR. 2020. https://doi.org/10. 1038/s41443-020-00377-5. [Epub ahead of print].

5. Sinatti C, Waterschoot M, Roth J, Van Laecke E, Hoebeke P, Spinoit AF. Long-term sexual outcomes in patients with exstrophy-epispadias complex. IJIR. 2020. https://doi.org/10. 1038/s41443-020-0248-2. [Epub ahead of print].

6. Banuelos B, Hiess M, Stein R, Gonzales R, Lingau A, Wood D, et al. Sexual function in adult patients who have undergone augmentation surgery in childhood: what is really important? IJIR. 2020. https://doi.org/10.1038/s41443-020-00355-x. [Epub ahead of print].

7. Sinatti C, Wolff D, Buncamper M, Verla W, Claes K, Lumen N, et al. Phalloplasty in cis-men with penile insufficiency: evaluation of outcomes and surgical complications. IJIR. 2020. https://doi. org/10.1038/s41443-020-00385-5. [Epub ahead of print].

8. Greear GM, Romano MF, Katz MH, Munarriz R, Rague JT. Testicular torsion: epidemiological risk factors for orchiectomy in pediatric and adult patients. IJIR. 2020. https://doi.org/10.1038/ s41443-020-0331-8. [Epub ahead of print].

9. Hofmann A, Vauth F, Roesch WH. Zinner syndrome and infertility - a literature review based on a clinical case. IJIR. 2020. https://doi.org/10.1038/s41443-020-00360-0. [Epub ahead of print].

10. Earp BD, Johnsdotter S. Current critiques of the WHO policy on female genital mutilation. IJIR. 2020. https://doi.org/10.1038/ s41443-020-0302-0. [Epub ahead of print].

11. Hiess M. Medical approach to children who may have been sexually abused-a narrative review: medical assessment in suspected sexual child abuse. IJIR. 2020. https://doi.org/10.1038/ s41443-020-00353-z. [Epub ahead of print].

12. Baetens L, Dhondt K. Psychosocial challenges and hormonal treatment in gender diverse children and adolescents. A narrative review. IJIR. 2020. https://doi.org/10.1038/s41443-020-0291-z. [Epub ahead of print].

13. Lampalzer U, Briken P, Schweizer K. Psychosocial care and counselling in the field of diverse sex development (dsd): parents' experiences, needs and improvements. IJIR. 2020. [Epub ahead of print]. 